Article

\title{
Multi-Objective Optimization of Fog Computing Assisted Wireless Powered Networks: Joint Energy and Time Minimization
}

\author{
Yuan Liu ${ }^{1,2}$, Ke Xiong ${ }^{1,2, * \mathbb{D}}$, Yu Zhang ${ }^{3,4}$, Li Zhou ${ }^{5, * \mathbb{D}}$, Fuhong Lin $\left.{ }^{4} \mathbb{(}\right)$ and Tong Liu ${ }^{6}$ \\ 1 School of Computer and Information Technology, Beijing Jiaotong University, Beijing 100044, China; \\ 17112090@bjtu.edu.cn \\ 2 Beijing Key Laboratory of Traffic Data Analysis and Mining, Beijing Jiaotong University, \\ Beijing 100044, China \\ 3 State Grid Energy Research Institute Co., Ltd., Beijing 102209, China; zhangyu2@sgeri.sgcc.com.cn \\ 4 School of Computer and Communication Engineering, University of Science and Technology, \\ Beijing 100083, China; FHLin@ustb.edu.cn \\ 5 School of Information, Beijing Wuzi University, Beijing 101149, China \\ 6 Beijing Computing Center, Beike Industry Park, Beijing 100094, China; Liutong@bcc.ac.cn \\ * Correspondence: kxiong@bjtu.edu.cn (K.X.); zhoulibit@126.com (L.Z.); Tel.: +86-010-51688536 (K.X.)
}

Received: 25 December 2018; Accepted: 24 January 2019; Published: 29 January 2019

\begin{abstract}
This paper studies the optimal design of the fog computing assisted wireless powered network, where an access point (AP) transmits information and charges an energy-limited sensor device with Radio Frequency (RF) energy transfer. The sensor device then uses the harvested energy to decode information and execute computing. Two candidate computing modes, i.e., local computing and fog computing modes, are considered. Two multi-objective optimization problems are formulated to minimize the required energy and time for the two modes, where the time assignments and the transmit power are jointly optimized. For the local computing mode, we obtain the closed-form expression of the optimal time assignment for energy harvesting by solving a convex optimization problem, and then analyze the effects of scaling factor between the minimal required energy and time on the optimal time assignment. For the fog computing mode, we derive closed-form and semi-closed-form expressions of the optimal transmit power and time assignment for offloading by adopting the Lagrangian dual method, the Karush-Kuhn-Tucker (KKT) conditions and Lambert $\mathcal{W}$ Function. Simulation results show that, when the sensor device has poor computing capacity or when it is far away from the AP, the fog computing mode is the better choice; otherwise, the local computing is preferred to achieve a better performance.
\end{abstract}

Keywords: local computing; fog computing; SWIPT; multi-objective; convex optimization

\section{Introduction}

Recent advancements in the fifth-generation (5G) have enabled abundant technologies such as vehicular networks, autonomous control, and Internet of Things (IoT) [1-3]. Many sensors are distributed in IoT, and they need to collect many data and execute real-time communication tasks, which are quite latency-sensitive. However, due to their small sizes and limited communication, computation, and storage resources, it is difficult for sensor devices to complete computation tasks themselves.

To enhance wireless sensors' computation capabilities, cloud computing was presented as an effective technology, with which sensor devices could offload computational-intensive tasks to cloud servers for computing [4-6]. Nevertheless, traditional central clouds are usually remotely located 
and far away from wireless sensor devices, thus offloading computation tasks to centralized cloud servers may results in heavy access burden and transmission delay. Therefore, a new communication and computing paradigm called fog computing was presented to extend cloud computing from network center to network edge $[7,8]$. Compared with cloud servers, fog servers are closer to user terminal equipments. Thus, fog computing is able to achieve lower response delay. Moreover, by offloading computation intensive tasks from ultra-low-power sensor devices to fog servers, the energy consumption of sensors may be saved and computation capabilities of wireless sensors is capable of being supplemented.

Apart from the limited computation capacity of ultra-low-power sensor devices, another key issue is how to supply sustainable and stable power to sensor devices, because IoT sensor devices are often widely deployed in large-scale networks and powered by small-size batteries with limited energy storage capacities. To release the cost and risk of battery replacement, in large-scale applications and toxic environment, wireless power transfer (WPT) was presented as a promising solution. As for wireless power transfer, there are three different kinds of technologies, i.e., induction coupling, magnetic resonance coupling and RF radiation. Among them, induction coupling and magnetic resonance coupling are near-field power transfer technologies, which basically are able to charge the devices in the range of tenths of watts, over short distances of up to one meter [9]. Particularly, induction coupling needs tight alignment of the coils of chargers and charging devices [10], and magnetic resonance coupling needs magnetic nuclei, external magnetic field and radio frequency magnetic field [11]. As for RF radiation, it is a far-field wireless power transfer technology, which is capable of transferring power in the range of several milliWatts at a distance of up to several meters [12,13]. Although its energy transfer efficiency is relatively low due to the path loss, it does not require the tight alignment of the coils and resonance. Moreover, harvesting energy from man-generated RF sources is controllable and relatively reliable. Thus, RF-based EH is suitable for low-power devices and is considered as a promising solution to power the IoTs and wireless sensor networks (WSNs) by deploying dedicated power stations [14-16].

RF-based EH has two main application paradigms in wireless communications, i.e., wireless powered communication networks (WPCNs) and simultaneous wireless information and power transfer (SWIPT). In WPCNs, sensor devices harvest energy from RF signal at first, and then use the harvested energy for information transmission. In SWIPT, energy and information are carried by the same transmitted signals. Thus far, two types of SWIPT receiver architectures, namely power splitting (PS) [17-20] and time switching (TS) [20-22] receivers, have been extensively investigated. With PS architecture, the received signal is divided into two streams, where one stream is used for EH, and the other is used for information decoding (ID). With TS architecture, the receiver can switch the operation between information decoding and energy harvesting over time.

\subsection{Related Works}

Thus far, fog computing and RF-based EH have been widely studied for wireless networks, where, however, most early works study them separately. For example, in [23,24], the computation latency was minimized with dynamic arriving tasks and channel fading in single-user MEC systems. In [25], the computation and communication resources allocation at both the user and the helper were optimized to minimize their total energy consumption for partial and binary offloading modes. In [26,27], the energy-efficient design in multiuser MEC system was studied with the objective to minimize the users' energy consumption. However, these works did not involve RF-based EH technology. In [28], the sum-throughput of all users was maximized by jointly optimizing the time allocation for wireless power transfer and information transmissions in emerging wireless powered communication network. In [29], a novel cooperative SWIPT scheme was proposed and a resource allocation problem is formulated to maximize the network energy efficiency. However, the work in $[28,29]$ did not consider fog computing technology. 
Recently, to exploit the benefits of both fog computing and RF-based EH, a few works investigated wireless powered fog computing system to achieve self-sustainable computing for wireless sensor devices. In [30], a user cooperation approach was presented to improve the computation performance and maximize the computation rate by jointly optimizing the transmit energy beamforming, as well as the communication and computation resource allocations. In [31], the probability of successful computing was maximized for given data in a wireless powered MEC system. In [32], a unified MEC-WPT design was proposed to minimize the AP's total energy consumption. In [33], cooperative communication was considered to minimize the total transmit energy consumption in an energy-efficient (EE) wireless powered MEC system. In [34], the fundamental tradeoff between energy efficiency (EE) and delay was investigated by jointly optimizing computation allocation and resource management policy in a wireless powered MEC system. In [35], the weighted sum computation rate was maximized by jointly optimizing the individual computing mode selection and the transmission time allocation in a multi-user MEC powered network.

\subsection{Motivations}

In this paper, we also investigate a fog computing assisted wireless powered network. In such a network, since the energy, communication and computation resources are closely coupled together, how to efficiently fuse them is very essential for the system. Thus far, many fundamental laws on designing fog computing assisted wireless powered networks have not been revealed yet. Motivated by this, this paper studies a typical three-node fog computing SWIPT network model, in which an ultra-low-power sensor adopts TS SWIPT architecture to harvest energy from the AP and then completes computation-intensive tasks. Our goal is to complete a given computation task with as little energy and time as possible. Two main differences between this paper and existing ones are summarized as follows.

First, compared to most existing works, which only study single-objective optimization problems, we study a multi-objective optimization problem. For example, in $[23,24]$, their goals were only to minimize the system delay. In [36], the goal was to minimize the required power. In [37], the goal was to maximize the energy efficiency, and in [38], the goal was to maximize the end-to-end information rate. Considering that, in practical applications, multiple performance indexes need to be minimized/maximized at the same time, multi-objective optimization problems are established to minimize the required energy and time in this paper rather than the single objective design.

Second, most studies aim at maximizing the information transmission and communication capacity of the system for given energy and time resources to explore the system transmission and computing capacity. In this paper, we desire to explore the minimal required energy and time resources to complete a given information task, which caters for the requirement of future energy efficient green communication and computing system design.

\subsection{Contributions}

In this paper, to obtain some fundamental laws for such kind of systems, a typical three-node system model is studied, in which a ultra-low-power sensor device adopts TS SWIPT architecture to harvest energy from the AP and then completes the computation-intensive tasks. Due to its limited energy and computing capability, the sensor may not be able to complete the computational task itself, so it can offload the task to the fog server for computing. For such a typical SWIPT-enabled fog computing system, the main contributions of this paper are summarized as follows.

(1) Considering that both energy and time are very important performance matrices in wireless sensor networks, for a given task, we mathematically formulate two multi-objective optimization problems to minimize the required energy and time of the local computing and fog computing modes by jointly optimizing the time assignments for energy harvesting and offloading, and the transmit power at sensor device for offloading subject to system latency constraint and energy resource allocation constraint. 
(2) For the local computing mode, we obtain the closed-form expression of the optimal time assignment for energy harvesting by solving a convex optimization problem, and then analyze the effects of scaling factor $\gamma$ between the minimal required energy and time on the system behavior. When the scaling factor is larger than the harvested power, the system tends to consume less time to complete delay-sensitive task, and in this case the time assignment for energy harvesting should take the minimum value while ensuring that the task can be completed on time. Otherwise, the system desires to harvest more energy, and the time assignment for energy harvesting should take the maximum value. For the fog computing mode, we derive closed-form and semi-closed-form expressions of the optimal transmit power and time assignment for offloading by adopting the Lagrangian dual method, the Karush-Kuhn-Tucker (KKT) conditions and Lambert $\mathcal{W}$ Function.

(3) With the obtained optimal time allocations for energy harvesting and offloading, and the optimal transmit power at the sensor for offloading, we study the effects of different system parameters (i.e., the distance between $\mathrm{AP}$ and sensor and the logic operations per bit of the sensor) on the selection of computing modes. Simulation results show that, when the sensor device has poor computing capacity or when it is far away from the $\mathrm{AP}$, the fog computing should be selected to alleviate the computational burden and save energy for the sensor device.

\subsection{Organization}

The remainder of this paper is organized as follows. Section 2 introduces the system model of the wireless powered three-node fog computing system. Section 3 introduces two multi-objective optimization problems to minimize the required energy and time of the local computing and fog computing modes, respectively. Section 4 provides simulation results and numerical analysis. Finally, we conclude the paper in Section 5.

\section{System Model}

Consider a wireless powered network composed of a single-antenna AP, one single-antenna ultra-low-power sensor and a fog server. It is assumed that the sensor is energy-limited and has no available power to perform computing and communication operations, thus it requires harvesting energy from the transmitted signals of the AP using SWIPT technology. Once the sensor receives the information and harvests sufficient energy, it desires processing the information (or task) by computing. For the task processing, two computation modes are available, i.e., local computing and fog computing, as shown in Figure 1. That is, the sensor can process the task itself by using local computing mode or offload the task to the fog server for computing by using fog computing mode.

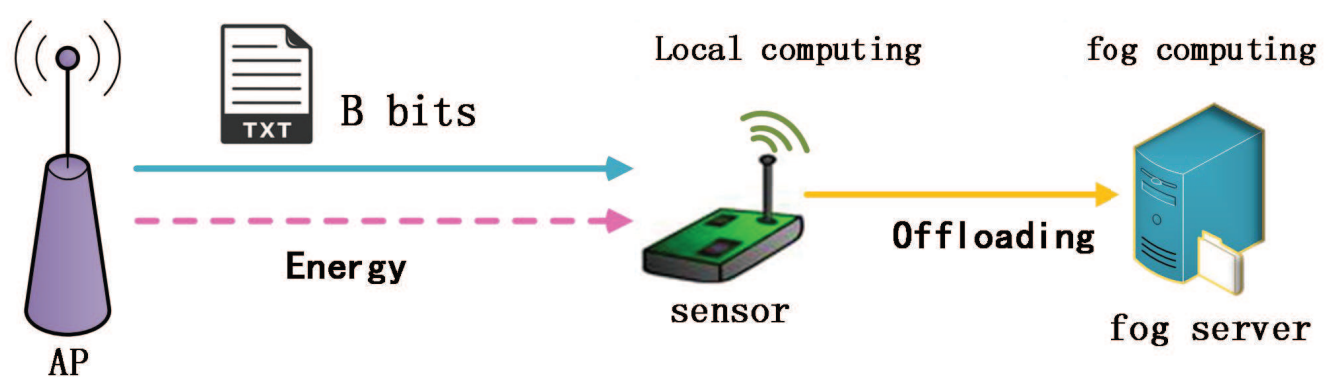

Figure 1. System model.

Block fading channel model is assumed, where the length of a time block is denoted with $T$. $T$ is chosen to be no longer than the coherence time of the channel so that the coefficients remain unchanged during $T$, but they may change from one block to the next following Rayleigh distribution. To fulfill the information transmission and task computing, as shown in Figure 2, $T$ is divided into three orthogonal time slots, where the first time slot is used for the sensor to harvest energy, the second 
time slot is used for the sensor to decode information, and the third time slot is used for the sensor to execute computing, i.e., local computing or fog computing.

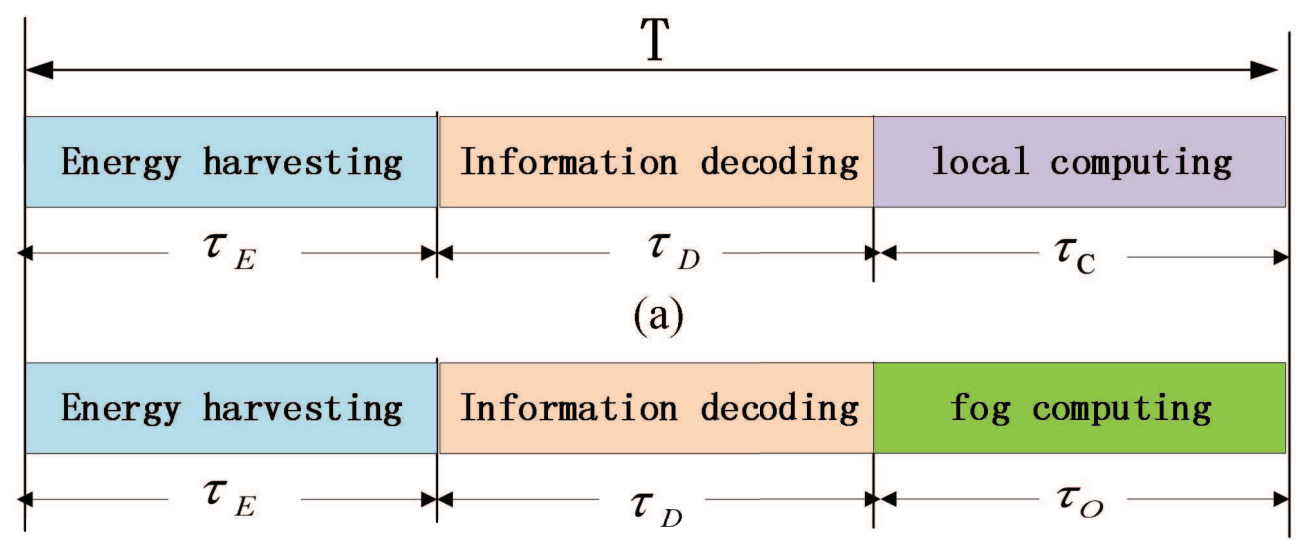

(b)

Figure 2. Time allocation frameworks: (a) local computing mode; and (b) fog computing mode.

Specifically, in the first time slot with time interval $\tau_{E}$, AP transfers energy to the sensor device, and the harvested energy at the sensor over this block can be given by

$$
E_{H}=\eta P_{1}\left|h_{1}\right|^{2} \tau_{E}
$$

where $0<\eta \leq 1$ is the constant energy harvesting efficiency factor of the sensor, $P_{1}$ is $\mathrm{AP}^{\prime} \mathrm{s}$ transmit power, and $h_{1}$ is the channel power gain from the AP to the sensor.

In the second time slot with time interval $\tau_{D}$, AP transmits a certain amount of data to the sensor, and the available transmission rate $R_{1}($ bits $/ s)$ is

$$
R_{1}=w_{1} \log _{2}\left(1+\frac{P_{1}\left|h_{1}\right|^{2}}{w_{1} \sigma_{0}^{2}}\right)
$$

where $w_{1}$ is the communication bandwidth and $\sigma_{0}^{2}$ is the noise power of unit bandwidth at the sensor. Assume that the computation task is with $B$ input bits, where $B \geq 0$. According to (2), the information transmission time interval $\tau_{D}$ is

$$
\tau_{D}=\frac{B}{w_{1} \log _{2}\left(1+\frac{P_{1}\left|h_{1}\right|^{2}}{w_{1} \sigma_{0}^{2}}\right)} .
$$

Since the amount of decoding energy consumption per bit depends on the decoding method, e.g., analog or digital techniques and the CMOS circuit design [39], the decoding energy consumption can be modeled by

$$
E_{D}=\varepsilon B,
$$

where $\varepsilon$ (Joule/bit) is a constant depending on the technology and circuit design.

In the third time slot, the sensor device processes the task itself or offloads the task to the fog server for computing. If the sensor choose the local computing mode, the execution time can be given by

$$
\tau_{\mathrm{C}}=\frac{K B}{f_{m}}
$$

where $f_{m}$ is the computation capability, i.e., the CPU clock speed (Cycles/s) of the sensor. Moreover, according to [40], the minimum dynamic switching energy per logic gate is $C_{g} V_{D D}^{2}$, where $C_{g}$ is the 
gate input capacitance and $V_{D D}$ is the supply voltage. This energy consumption is estimated with the Landauer limit, i.e., $M_{c} N_{0} \ln (2)$ [41], where $M_{c}$ is a time-dependent immaturity factor of the technology and $N_{0}$ is the thermal noise spectral density. Therefore, the computation energy consumption of the local computing mode is modeled by

$$
E_{C}=F_{0} \alpha M_{c} N_{0} \ln (2) K B,
$$

where $F_{0}$ is the fanout, which is the number of loading logic gates, typically with values of $3-4, \alpha$ is the activity factor typically with values of $0.1-0.2, K$ is the number of logic operations per bit and $B$ is the number of received bits of the sensor device during the block.

If the fog computing mode is selected, the sensor offloads computation task to the fog server for computing, and the available transmission rate $R_{2}(b i t s / s)$ from the sensor to the fog server is

$$
R_{2}=w_{2} \log _{2}\left(1+\frac{P_{O}\left|h_{2}\right|^{2}}{w_{2} \sigma_{0}^{2}}\right)
$$

where $w_{2}$ is the communication bandwidth, $h_{2}$ is the channel power gain between the sensor device and fog server, and $P_{O}$ denotes the transmit power of the sensor device for offloading. Correspondingly, the offloading time with $B$ bits can be given by

$$
\tau_{O}=\frac{B}{w_{2} \log _{2}\left(1+\frac{P_{O}\left|h_{2}\right|^{2}}{w_{2} \sigma_{0}^{2}}\right)} .
$$

Then, the required energy for offloading the computation task to the fog server can be given by

$$
E_{O}=\tau_{O} P_{O}
$$

Due to the sufficient energy and superior computation and communication capabilities of the fog server, similar to many existing works $[28,33]$, we also neglect the time for fog server to execute computation task and feed back the computation results to the sensor. Since sensor needs to accomplish task execution within a block, the system latency is limited by the block length $T$, i.e.,

$$
\tau_{E}+\tau_{D}+I_{O} \tau_{C}+\left(1-I_{O}\right) \tau_{O} \leq T
$$

where $I_{O}$ is an indicator variable, with $I_{O}=1$ indicating the local computing mode being selected and $I_{O}=0$ indicating the fog computing mode being selected. Besides, in each mode, the energy consumed at the sensor could not exceed the harvested energy $E_{H}$ during per block, thus it satisfies

$$
E_{D}+I_{O} E_{C}+\left(1-I_{O}\right) E_{O} \leq E_{H}
$$

\section{Optimal Resource Allocation and Offloading Decision}

For the described fog computing assisted WPCN networks, this section formulates a multi-objective optimization problem to minimize the required energy and time to complete the computation task under two computation modes (i.e., local computing or offloading) by jointly optimizing the time allocation for energy harvesting and offloading, and the transmit power for offloading of the sensor. 


\subsection{Local Computing Mode}

The optimization problem is mathematically given by

$$
\begin{aligned}
& \mathbf{P}_{\mathbf{A}_{0}}: \min _{\tau_{E}} E_{D}+E_{C}-E_{H} \\
& \min _{\tau_{E}} \tau_{D}+\tau_{C}+\tau_{E} \\
& \text { s.t. } C_{1}: E_{D}+E_{C} \leq E_{H} \\
& C_{2}: \tau_{D}+\tau_{C}+\tau_{E} \leq T
\end{aligned}
$$

where $C_{1}$ of $\mathbf{P}_{\mathbf{A}_{0}}$ indicates that decoding energy consumption and local computing energy consumption are limited by the harvested energy per block, and $C_{2}$ of $\mathbf{P}_{\mathbf{A}_{0}}$ is the sensor's latency constraint. To make the original problem more tractable, we establish a multi-objective optimization new mathematics model by introducing a new variable $\gamma$ (joule/s), which is defined as a scaling factor between the minimal required energy and time. Then, we analysis the optimal value of optimization variable for energy harvesting when $\gamma$ takes different values, i.e., energy consumption and time occupying different proportions in the objective function.

$$
\begin{aligned}
\mathbf{P}_{\mathbf{A}}: & \min _{\tau_{E}} E_{D}+E_{C}-E_{H}+\gamma\left(\tau_{D}+\tau_{C}+\tau_{E}\right) \\
\text { s.t. } & C_{1}: E_{D}+E_{C} \leq E_{H} \\
& C_{2}: \tau_{D}+\tau_{C}+\tau_{E} \leq T \\
& C_{3}: \gamma \geq 0
\end{aligned}
$$

To better unearth the characteristics of problem $\mathbf{P}_{\mathbf{A}}$, by expanding the expressions of the corresponding variables, the problem can be expressed by

$$
\begin{array}{ll}
\mathbf{P}_{\mathbf{A}_{1}}: & \min _{\tau_{E}} \varepsilon B+F_{0} \alpha M_{c} N_{0} \ln (2) K B-\eta P_{1}\left|h_{1}\right|^{2} \tau_{E}+\gamma\left(\frac{B}{w_{1} \log _{2}\left(1+\frac{P_{1}\left|h_{1}\right|^{2}}{w_{1} \sigma_{0}^{2}}\right)}+\frac{K B}{f_{m}}+\tau_{E}\right) \\
\text { s.t. } & C_{1}: \varepsilon B+F_{0} \alpha M_{c} N_{0} \ln (2) K B \leq \eta P_{1}\left|h_{1}\right|^{2} \tau_{E} \\
& C_{2}: \tau_{E}+\frac{B}{w_{1} \log _{2}\left(1+\frac{P_{1}\left|h_{1}\right|^{2}}{w_{1} \sigma_{0}^{2}}\right)}+\frac{K B}{f_{m}} \leq T \\
& C_{3}: \gamma \geq 0
\end{array}
$$

It can be seen that, in the objective function, the constraints $C_{1}$ and $C_{2}$ of problem $\mathbf{P}_{\mathbf{A}_{1}}$ are linear functions of $\tau_{E}$, thus $\mathbf{P}_{\mathbf{A}_{1}}$ is a convex optimization problem, which can be readily solved by using the optimization toolbox cvx [42].

Proposition 1. The optimal time assignment for energy harvesting $\tau_{E}^{*}$ of $\boldsymbol{P}_{A_{1}}$ is

$$
\tau_{E}^{*}=\left\{\begin{aligned}
\frac{E_{D}+E_{C}}{\eta P_{1}\left|h_{1}\right|^{2}}, & \text { if } \gamma \geq \eta P_{1}\left|h_{1}\right|^{2} \\
T-\tau_{D}-\tau_{C}, & \text { otherwise }
\end{aligned}\right.
$$

Proof of Proposition 1. By combining the terms of optimization variable $\tau_{E}$ in the objective function of $\mathbf{P}_{\mathbf{A}_{1}}$, we can get a new term $\left(\gamma-\eta P_{1}\left|h_{1}\right|^{2}\right) \tau_{E}$, which is a positive value when $\gamma \geq \eta P_{1}\left|h_{1}\right|^{2}$. It decreases as $\tau_{E}$ decreases, $\tau_{E}$ should take the minimum value to minimize it, and constraint $C_{1}$ of $\mathbf{P}_{\mathbf{A}_{1}}$ gives the lower bound of $\tau_{E}$ when $C_{1}$ takes the equation. Otherwise, $\left(\gamma-\eta P_{1}\left|h_{1}\right|^{2}\right) \tau_{E}$ is a negative value, it decreases as $\tau_{E}$ increases, and $\tau_{E}$ should take the maximum value to minimize it. Constraint $C_{2}$ of $\mathbf{P}_{\mathbf{A}_{1}}$ gives the upper bound of $\tau_{E}$ when $C_{2}$ takes the equation.Hence, Proposition 1 is proved. 


\subsection{Fog Computing Mode}

In this section, we formulate a multi-objective optimization problem to minimize the energy consumption and time subject to the energy consumption constraint and delay constraint by jointly optimizing the time assignment for energy harvesting and offloading and the sensor's transmit power for offloading, which is mathematically given by

$$
\begin{array}{ll}
\mathbf{P}_{\mathbf{B}_{0}}: \min _{\tau_{E}, P_{O}, \tau_{O}} E_{D}+E_{O}-E_{H} \\
& \min _{\tau_{E}, P_{O}, \tau_{O}} \tau_{D}+\tau_{O}+\tau_{E} \\
\text { s.t. } & C_{1}: \tau_{O} w_{2} \log _{2}\left(1+\frac{P_{O}\left|h_{2}\right|^{2}}{w_{2} \sigma_{0}^{2}}\right) \geq B \\
& C_{2}: E_{D}+E_{O} \leq E_{H} \\
& C_{3}: \tau_{D}+\tau_{O}+\tau_{E} \leq T \\
& C_{4}: \gamma \geq 0
\end{array}
$$

where $\mathrm{C}_{1}$ of $\mathbf{P}_{\mathbf{B}_{0}}$ indicates the required minimum information transmission rate of the sensor device to finish offloading, $\mathrm{C}_{2}$ of $\mathbf{P}_{\mathbf{B}_{0}}$ indicates that the energy consumption for information decoding and offloading of the sensor device can not exceed the harvested energy during per block, and $\mathrm{C}_{3}$ of $\mathbf{P}_{\mathbf{B}_{0}}$ is the latency constraint of the sensor. Similar to the process of $\mathbf{P}_{\mathbf{A}_{0}}$, we also introduce $\gamma$ to re-express the problem $\mathbf{P}_{\mathbf{B}_{0}}$, which is given by

$$
\begin{aligned}
\mathbf{P}_{\mathbf{B}}: & \min _{\tau_{E}, P_{O}, \tau_{O}} E_{D}+E_{O}-E_{H}+\gamma\left(\tau_{D}+\tau_{O}+\tau_{E}\right) \\
\text { s.t. } & C_{1}: \tau_{O} w_{2} \log _{2}\left(1+\frac{P_{O}\left|h_{2}\right|^{2}}{w_{2} \sigma_{0}^{2}}\right) \geq B \\
& C_{2}: E_{D}+E_{O} \leq E_{H} \\
C_{3}: & \tau_{D}+\tau_{O}+\tau_{E} \leq T \\
& C_{4}: \gamma \geq 0
\end{aligned}
$$

To better solve this problem, by expanding the expressions of $E_{D}, E_{O}, E_{H}, \tau_{D}$, and $\tau_{O}, \mathbf{P}_{\mathbf{B}_{0}}$ is further expressed by

$$
\begin{aligned}
& \mathbf{P}_{\mathbf{B}_{1}}: \min _{\tau_{E}, P_{O}, \tau_{O}} \varepsilon B+\tau_{O} P_{O}-\eta P_{1}\left|h_{1}\right|^{2} \tau_{E}+\gamma\left(\frac{B}{w_{1} \log _{2}\left(1+\frac{P_{1}\left|h_{1}\right|^{2}}{w_{1} \sigma_{0}^{2}}\right)}+\tau_{O}+\tau_{E}\right) \\
& \text { s.t. } C_{1}: \tau_{O} w_{2} \log _{2}\left(1+\frac{P_{O}\left|h_{2}\right|^{2}}{w_{2} \sigma_{0}^{2}}\right) \geq B \\
& \quad C_{2}: \varepsilon B+\tau_{O} P_{O} \leq \eta P_{1}\left|h_{1}\right|^{2} \tau_{E} \\
& \mathrm{C}_{3}: \frac{B}{w_{1} \log _{2}\left(1+\frac{P_{1}\left|h_{1}\right|^{2}}{w_{1} \sigma_{0}^{2}}\right)}+\tau_{O}+\tau_{E} \leq T \\
& \mathrm{C}_{4}: \gamma \geq 0
\end{aligned}
$$

Problem $\mathbf{P}_{\mathbf{B}_{1}}$ is a non-convex problem because of the coupled variables of $\tau_{O}$ and $P_{O}$ in the objective function and constraint $C_{1}$. To decouple the non-convex problem, we define a new variable $\lambda$ $=P_{O} \tau_{O}$. By dosing so, $\mathbf{P}_{\mathbf{B}_{1}}$ is re-expressed by

$$
\begin{aligned}
\mathbf{P}_{\mathbf{B}_{2}} & : \min _{\tau_{E}, \lambda, \tau_{O}} \varepsilon B+\lambda-\eta P_{1}\left|h_{1}\right|^{2} \tau_{E}+\gamma\left(\frac{B}{w_{1} \log _{2}\left(1+\frac{P_{1}\left|h_{1}\right|^{2}}{w_{1} \sigma_{0}^{2}}\right)}+\tau_{O}+\tau_{E}\right) \\
\text { s.t. } C_{1}: & : \tau_{O} w_{2} \log _{2}\left(1+\frac{\lambda\left|h_{2}\right|^{2}}{\tau_{O} w_{2} \sigma_{0}^{2}}\right) \geq B \\
C_{2}: & \varepsilon B+\lambda \leq \eta P_{1}\left|h_{1}\right|^{2} \tau_{E} \\
C_{3} & : \frac{B}{w_{1} \log _{2}\left(1+\frac{P_{1}\left|h_{1}\right|^{2}}{w_{1} \sigma_{0}^{2}}\right)}+\tau_{O}+\tau_{E} \leq T \\
C_{4} & : \gamma \geq 0
\end{aligned}
$$


Then, the constraint $C_{1}$ of $\mathbf{P}_{\mathbf{B}_{2}}$ satisfies the form of the projection function, i.e., $x \log \left(1+\frac{y}{x}\right)$, which is concave with respect to $x$ and $y$, so the left side of $C_{1}$ is concave of both $\lambda$ and $\tau_{O}$. Thus, problem $\mathbf{P}_{\mathbf{B}_{2}}$ can be transformed into $\mathbf{P}_{\mathbf{B}_{3}}$, i.e., the Lagrangian function of the problem $\mathbf{P}_{\mathbf{B}_{2}}$, which is given by

$$
\begin{aligned}
\mathbf{P}_{\mathbf{B}_{3}} & : \zeta\left(\lambda, \tau_{E}, \tau_{O}, \mu, \vartheta, v\right)=\varepsilon B+\lambda-\eta P_{1}\left|h_{1}\right|^{2} \tau_{E}+\gamma\left(\frac{B}{w_{1} \log _{2}\left(1+\frac{P_{1}\left|h_{1}\right|^{2}}{w_{1} \sigma_{0}^{2}}\right)}+\tau_{O}+\tau_{E}\right) \\
& -\mu\left(\tau_{O} w_{2} \log _{2}\left(1+\frac{\lambda\left|h_{2}\right|^{2}}{\tau_{O} w_{2} \sigma_{0}^{2}}\right)-B\right) \\
& -\vartheta\left(\eta P_{1}\left|h_{1}\right|^{2} \tau_{E}-\varepsilon B-\lambda\right) \\
& -v\left(T-\tau_{E}-\tau_{D}-\tau_{O}\right)
\end{aligned}
$$

where $\mu, \vartheta, v$ are the Lagrange multipliers corresponding to constraints $C_{1}, C_{2}$ and $C_{3}$, and the dual problem of problem $\mathbf{P}_{\mathbf{B}_{3}}$ is

$$
\begin{aligned}
& \max _{\mu, \vartheta, v} \min _{\tau_{E}, \lambda, \tau_{O}} \zeta \\
& \text { s.t. } \mu \geq 0, \vartheta \geq 0, v \geq 0
\end{aligned}
$$

The Karush-Kuhn-Tucker (KKT) conditions are necessary and sufficient conditions to obtain optimal solutions, and the KKT conditions for $\mathbf{P}_{\mathbf{B}_{3}}$ with respect to the optimal solutions of $\tau_{E^{*}}^{*} \lambda^{*}, \tau_{O}^{*}$ are as listed in Equation (20).

$$
\left\{\begin{array}{l}
\mu^{*} \geq 0, \quad \vartheta^{*} \geq 0, \quad v^{*} \geq 0 \\
\mu\left(\tau_{O} w_{2} \log _{2}\left(1+\frac{\lambda\left|h_{2}\right|^{2}}{\tau_{O} w_{2} \sigma_{0}^{2}}\right)-B\right)=0 \\
\vartheta\left(\eta P_{1}\left|h_{1}\right|^{2} \tau_{E}-\varepsilon B-\lambda\right)=0 \\
v\left(T-\tau_{D}-\tau_{E}-\tau_{O}\right)=0 \\
\tau_{O} w_{2} \log _{2}\left(1+\frac{\lambda\left|h_{2}\right|^{2}}{\tau_{O} w_{2} \sigma_{0}^{2}}\right) \geq B \\
\varepsilon B+\lambda \leq \eta P_{1}\left|h_{1}\right|^{2} \tau_{E} \\
\tau_{D}+\tau_{O}+\tau_{E} \leq T, \\
\frac{\partial \zeta}{\partial \tau_{E}^{*}}=0, \frac{\partial \zeta}{\partial \lambda^{*}}=0, \frac{\partial \zeta}{\partial \tau_{O}^{*}}=0
\end{array}\right.
$$

By expanding Equation (20h), one can obtain the following equations

$$
\begin{gathered}
\mu=\frac{\ln 2(1+\vartheta)}{w_{2}}\left(\frac{w_{2} \sigma_{0}^{2}}{\left|h_{2}\right|^{2}}+\frac{\lambda}{\tau_{O}}\right), \\
v=(1+\vartheta)\left(\eta P_{1}\left|h_{1}\right|^{2}\right)-\gamma,
\end{gathered}
$$

and

$$
w_{2} \mu\left[\log _{2}\left(1+\frac{\lambda\left|h_{2}\right|^{2}}{\tau_{O} w_{2} \sigma_{0}^{2}}\right)-\frac{\left.\lambda h_{2}\right|^{2}}{\left(\tau_{O} w_{2} \sigma_{0}^{2}+\lambda\left|h_{2}\right|^{2}\right) \ln 2}\right]=\gamma+v .
$$

By substituting the expressions of $\mu$ in (21) and $v$ in Equation (22) into Equation (23), one can obtain the following equation, i.e.,

$$
\ln 2\left(\frac{w_{2} \sigma_{0}^{2}}{\left|h_{2}\right|^{2}}+\frac{\lambda}{\tau_{O}}\right)\left[\log _{2}\left(1+\frac{\lambda}{\tau_{O}} \frac{\left|h_{2}\right|^{2}}{w_{2} \sigma_{0}^{2}}\right)-\frac{\frac{\lambda}{\tau_{O}} \frac{\left|h_{2}\right|^{2}}{w_{2} \sigma_{0}^{2}}}{\left(\frac{\lambda}{\tau_{O}} \frac{\left|h_{2}\right|^{2}}{w_{2} \sigma_{0}^{2}}+1\right) \ln 2}\right]=\eta P_{1}\left|h_{1}\right|^{2} .
$$


To simplify the above equation, by defining $\eta P_{1}\left|h_{1}\right|^{2}=\phi, \frac{\lambda}{\tau_{O}}=\psi$ and $\frac{\left|h_{2}\right|^{2}}{w_{2} \sigma_{0}^{2}}=\varepsilon$, Equation (24) can be simplified to be

$$
\ln 2\left(\frac{1}{\varepsilon}+\psi\right)\left[\log _{2}(1+\psi \varepsilon)-\frac{\psi \varepsilon}{(\psi \varepsilon+1) \ln 2}\right]=\phi .
$$

Proposition 2. The close-form expression of $\psi$ can be given by

$$
\psi=\frac{\phi \varepsilon-1-\mathcal{W}\left((\phi \varepsilon-1) \frac{1}{e}\right)}{\mathcal{W}\left((\phi \varepsilon-1) \frac{1}{e}\right) \varepsilon} .
$$

Proof of Proposition 2. According to Equation (25), we can get the close-form expression of $\psi$ shown in Equation (26). The derivation process is as follows:

$$
\begin{array}{rlr}
\ln 2\left(\frac{1}{\varepsilon}+\psi\right)\left[\log _{2}(1+\psi \varepsilon)-\frac{\psi \varepsilon}{(\psi \varepsilon+1) \ln 2}\right]=\phi, & \Leftrightarrow & (1+\psi \varepsilon) \log _{2}(1+\psi \varepsilon)=\frac{(\psi+\phi) \varepsilon}{\ln 2}, \Leftrightarrow \\
(1+\psi \varepsilon) \ln (1+\psi \varepsilon)=(\psi+\phi) \varepsilon, & \Leftrightarrow & (1+\psi \varepsilon) \ln (1+\psi \varepsilon)-(1+\psi \varepsilon)=\phi \varepsilon-1, \Leftrightarrow \\
\frac{e^{\ln (1+\psi \varepsilon)}}{e}[\ln (1+\psi \varepsilon)-1]=\frac{\phi \varepsilon-1}{e}, & \Leftrightarrow & (\ln (1+\psi \varepsilon)-1) e^{\ln (1+\psi \varepsilon)-1}=\frac{\phi \varepsilon-1}{e}, \Leftrightarrow \\
\ln (1+\psi \varepsilon)-1=\mathcal{W}\left(\frac{\phi \varepsilon-1}{e}\right), & \Leftrightarrow & \ln \left(\mathcal{W}\left(\frac{\phi \varepsilon-1}{e}\right)\right)=\ln \left(\frac{\phi \varepsilon-1}{e}\right)-\ln \left(\frac{1+\psi \varepsilon}{e}\right), \Leftrightarrow \\
\mathcal{W}(\phi \varepsilon-1) \frac{1}{e}=\frac{\phi \varepsilon-1}{1+\psi \varepsilon}, & \Leftrightarrow & \psi=\frac{\phi \varepsilon-1-\mathcal{W}\left((\phi \varepsilon-1) \frac{1}{e}\right)}{\mathcal{W}\left((\phi \varepsilon-1) \frac{1}{e}\right) \varepsilon},
\end{array}
$$

where $\mathcal{W}(\chi)$ is the Lambert $\mathcal{W}$ function with $\mathcal{W}(\chi) e^{\mathcal{W}(\chi)}=\chi$ [43]. Hence, Proposition 2 is proved.

Proposition 3. The optimal transmit power $p_{O}^{*}$ and time assignment $\tau_{O}^{*}$ for offloading are

$$
\begin{gathered}
p_{O}^{*}=\frac{\phi \varepsilon-1-\mathcal{W}\left((\phi \varepsilon-1) \frac{1}{e}\right)}{\mathcal{W}\left((\phi \varepsilon-1) \frac{1}{e}\right) \varepsilon}, \\
\tau_{O}^{*}=\frac{B}{w_{2} \log _{2}\left(1+\frac{P_{O}^{*}\left|h_{2}\right|^{2}}{w_{2} \sigma_{0}^{2}}\right)} .
\end{gathered}
$$

Proof of Proposition 3. According to the definition of $\lambda$ and $\psi$, one has that $\lambda^{*}=p_{O}^{*} \tau_{O}^{*}$ and $\psi=\frac{\lambda}{\tau_{O}}$, then $p_{O}^{*}=\psi$. According to Equation (21), the Lagrange multiplier $\mu$ cannot be 0 to satisfy the KKT conditions in Equation (20b), and the constraint $C_{1}$ of $\mathbf{P}_{\mathbf{B}_{2}}$ should take the equation. Hence, Proposition 3 is proved.

Proposition 4. The optimal time assignment for energy harvesting $\tau_{E}^{*}$ of $\boldsymbol{P}_{\boldsymbol{B}_{2}}$ is

$$
\tau_{E}^{*}=\left\{\begin{aligned}
\frac{E_{D}+\tau_{O}^{*} P_{O}^{*}}{\eta P_{1}\left|h_{1}\right|^{2}} & \text { if } \gamma \geq \eta P_{1}\left|h_{1}\right|^{2} \\
T-\tau_{D}-\tau_{O}^{*} & \text { otherwise }
\end{aligned}\right.
$$

Proof of Proposition 4. With the obtained optimization variables $P_{O}^{*}$ and $\tau_{O}^{*}$, by combining the terms of optimization variable $\tau_{E}$ in the objective function of $\mathbf{P}_{\mathbf{B}_{2}}$, we can get a new term $\left(\gamma-\eta P_{1}\left|h_{1}\right|^{2}\right) \tau_{E}$, which is a positive value when $\gamma \geq \eta P_{1}\left|h_{1}\right|^{2}$. It decreases as $\tau_{E}$ decreases, thus $\tau_{E}$ should take the minimum value to minimize it. Constraint $C_{2}$ of $\mathbf{P}_{\mathbf{B}_{2}}$ gives the lower bound of $\tau_{E}$ when $C_{2}$ takes 
the equation. Otherwise, $\left(\gamma-\eta P_{1}\left|h_{1}\right|^{2}\right) \tau_{E}$ is a negative value and decreases as $\tau_{E}$ increases, thus $\tau_{E}$ should take the maximum value to minimize it. Constraint $C_{3}$ of $\mathbf{P}_{\mathbf{B}_{2}}$ gives the upper bound of $\tau_{E}$ when $\mathrm{C}_{3}$ takes the equation. Hence, Proposition 4 is proved.

\section{Numerical Analysis}

In this section, we present numerical results to discuss the system performance associated with two modes, i.e., local computing and fog computing modes. The simulated system model is shown in Figure 1 , in which the transmit power at AP is set to be 1 watt, and the channel power gain $h_{1}$ from the AP to the sensor and $h_{2}$ from the sensor to the fog server are generated following Rician fading with the Rician factor to being $3.5 \mathrm{~dB}$. The path-loss of the channels is modeled by the International Telecommunication Union (ITU) indoor channel model as [44]: $L=20 \log f c+N \log d-28$, where $L$ (dB) is the total path loss, $f c=4 \mathrm{GHz}$ is the frequency of transmission, $d(\mathrm{~m})$ is the distance, and $N$ $=22$ is the distance power loss coefficient. The system transmission bandwidth of both channels are set to be $w_{1}=w_{2}=1 \mathrm{MHz}$ and the noise power of unit bandwidth is $\sigma_{0}^{2}=-120 \mathrm{dBm}$. The energy harvesting efficiency of the sensor is $\eta=0.6$ and the decoding energy consumption per bit is $\epsilon=1$ $\mathrm{pJ} / \mathrm{bit}$. The block length is set to be $T=1 \mathrm{~s}$. The maximum constant CPU frequency of the sensor is $f_{u}=1 \times 10^{12}$ operations per second. Additionally, $M c=10^{4}, \alpha=0.1$, and $F_{0}=3$ [41].

Figure 3 depicts the harvested energy and the minimal required energy to complete the computation task versus the distance between AP and sensor. The AP, sensor and fog server are positioned on a straight line, the distance between $\mathrm{AP}$ and sensor is $d_{P S}$, and the distance between sensor and fog server is $d_{S f}$. It can be seen that the minimal required energy for decoding and computation of local computing mode are unchanged as $d_{P S}$ increases because the required energy for decoding and local computing depends on the amount of computation task. The minimal required energy of fog computing gradually decreases because the required energy for offloading decreases with the decrement of $d_{S f}$. Besides, the harvested energy gradually decreases in the local computing and fog computing modes with the increment of $d_{P S}$, and the difference of harvested energy between the two computing modes is not very large, especially for a relatively large $d_{p s}$.

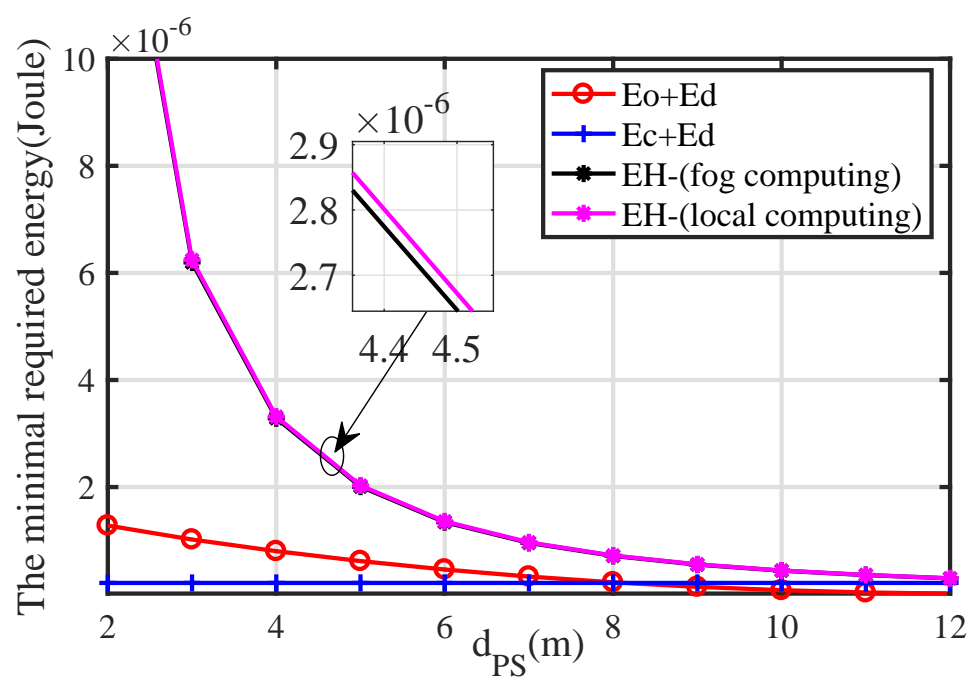

Figure 3. The minimal required energy and harvested energy versus $d_{P S}$.

Figure 4 depicts the effect of $d_{P S}$ on the computing modes selection of the sensor, where AP, sensor and fog server are located on a straight line, the location of AP and fog server are fixed, and the sensor moves away from the AP to the fog server. It can be seen that the minimal required energy of local computing mode is less than the minimal required energy of fog computing within a certain distance. 
It means that, in this case, local computing is more cost-effective; otherwise, when the sensor is close to the fog server, fog computing is more cost-effective.

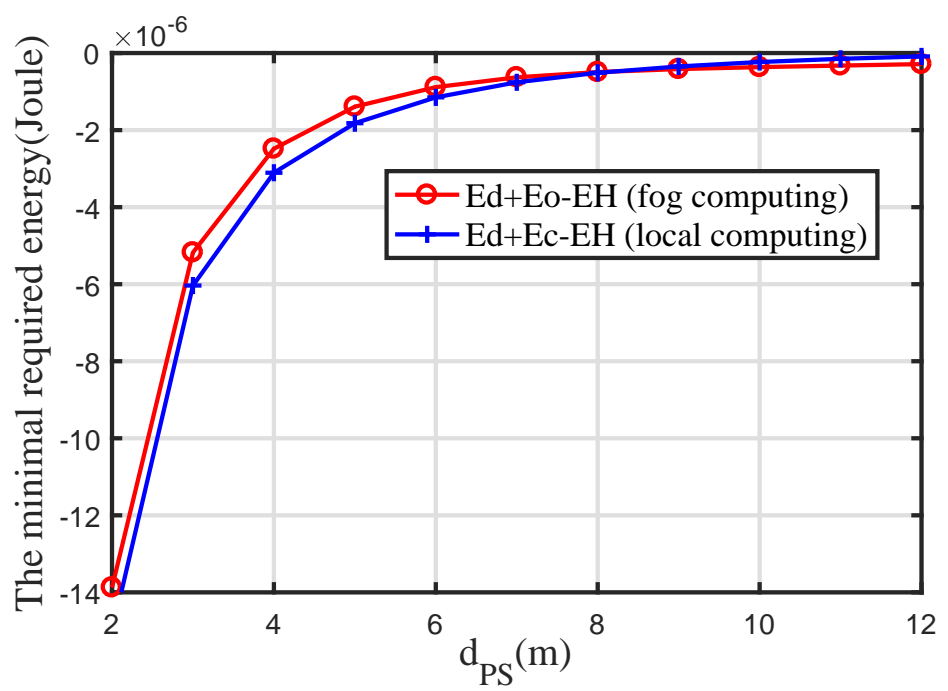

Figure 4. Performance comparison of the two computing modes versus $d_{P S}$.

Figure 5 depicts the minimal required time of two computing modes versus the distance $d_{P S}$ from $\mathrm{AP}$ to the sensor. It shows that the minimal required time of local computing mode increases because the harvested power and the achievable transmit information rate decreases with the increment of $d_{P S}$. Thus, it requires more time to harvest energy and decode information to complete the computation task. The minimal required time of fog computing mode increases firstly and then decreases because the required energy for offloading is firstly larger than the harvested power and then less than the harvested power with the increment of $d_{P S}$. Thus, the minimal required time for harvesting energy to complete the computation task firstly increases and then decreases. Besides, the required time for offloading also increases firstly and then decreases, and it is larger than local computing time.

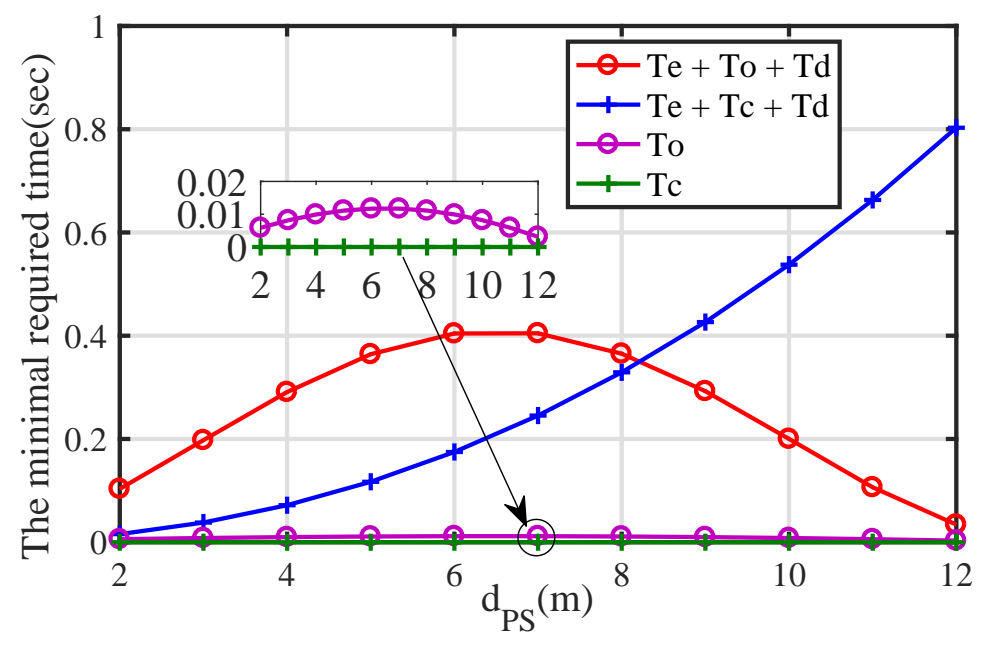

Figure 5. The minimal required time versus $d_{p s}$.

Figure 6 plots the minimal required energy of local computing and fog computing modes versus the number of operations per bit, $K$. It shows that the minimal required energy of local computing increases linearly with the increment of $K$, because the mathematical model of computation energy of 
local computing is linearly related to $K$. When $K$ is less than a specific value, the minimal required energy of local computing is less than fog computing, and the sensor may choose local computing mode; otherwise, when the sensor has poor computation capacity (i.e., $K$ is greater than a specific value), fog computing has better performance.

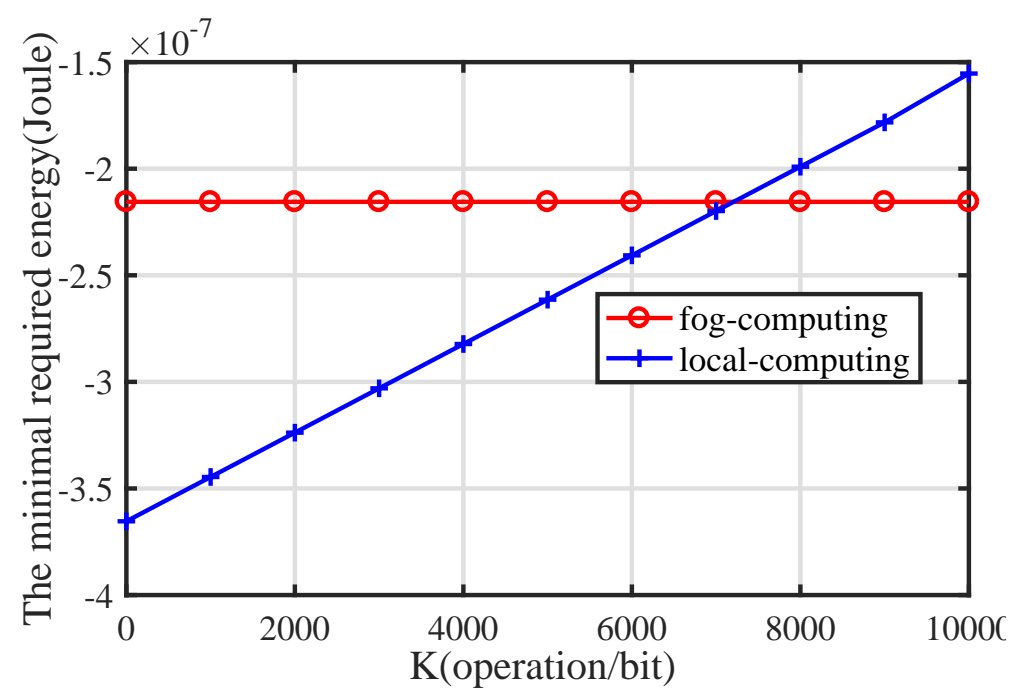

Figure 6. The minimal required energy versus $K$.

Figure 7 shows the performance comparison of two computing modes versus the position of the sensor on the 2-D coordinate plane, where the sensor moves on a $10 \times 10 \mathrm{~m}^{2}$ plane. The coordinates of the AP is $(-6,0)$, and the coordinates of the fog server is $(6,0)$. It shows that the local computing is more cost-effective when the sensor is within the light blue zone; otherwise, the fog computing mode has better performance when the sensor is located on the dark blue zone.

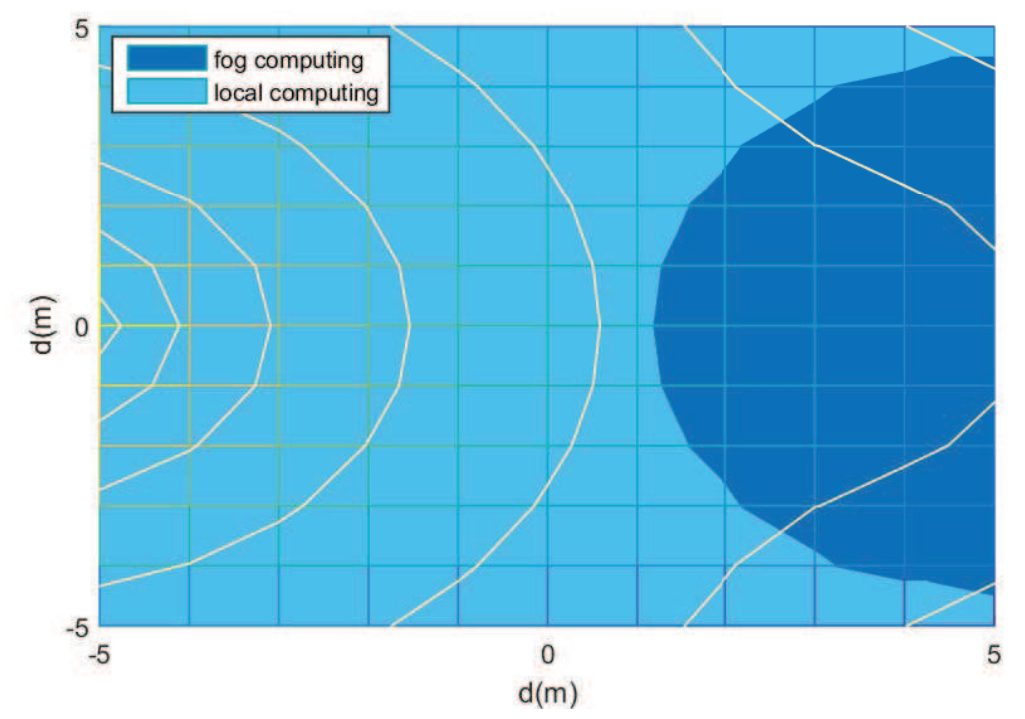

Figure 7. Performance comparison of two computing modes versus the position of the sensor on the 2-D coordinate plane.

Figure 8 plots the system performance versus $\gamma$, in which Figure 8 a plots the minimal required energy versus $\gamma$, Figure $8 \mathbf{b}$ plots the minimal required time versus $\gamma$, and Figure $8 \mathrm{c}$ plots the minimal required resources, i.e., the weighted sum of the minimal required energy and time versus $\gamma$. It can be seen that, when $\gamma$ is larger than the harvested power $6.6154 \times 10^{-7}$ (joule/s), the minimal required 
energy is almost equal to the harvested energy, and, in this case, the sensor tends to harvest just enough energy to complete delay-sensitive task. Otherwise, the minimal required time is almost equal to the length of a time block, and, in this case, the sensor desires to harvest more energy. Besides, the minimal required resources, i.e., the weighted sum of the minimal required energy and time, increases as $\gamma$ increases.

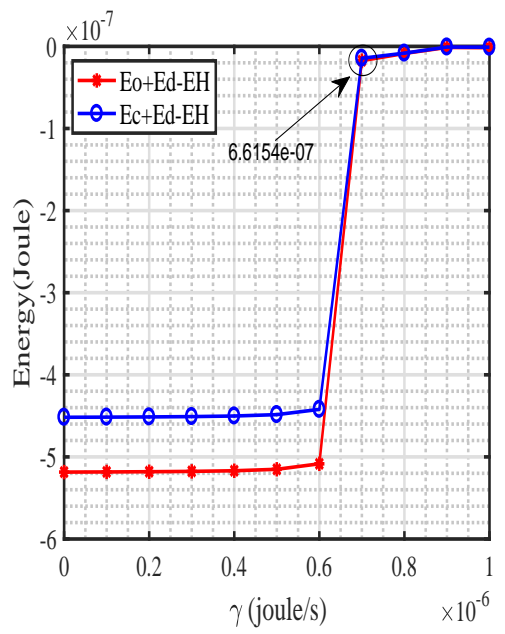

(a)

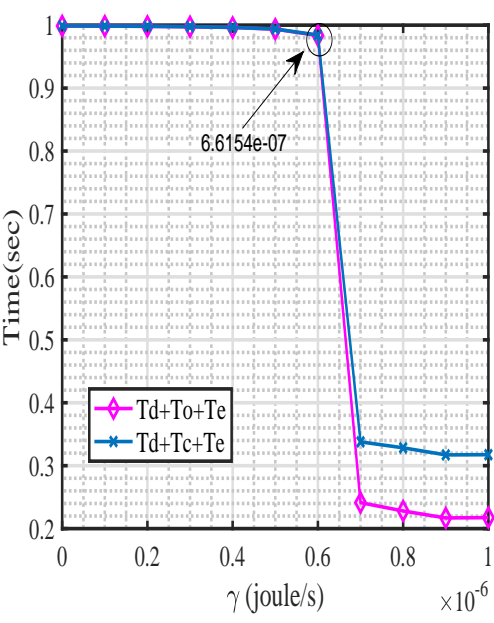

(b)

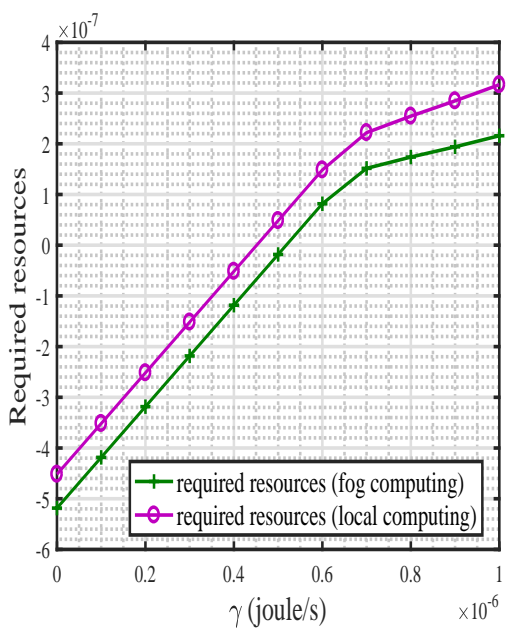

(c)

Figure 8. System performance versus $\gamma$.

\section{Conclusions}

We studied the optimal design of a fog computing assisted wireless powered network. We formulated two multi-objective optimization problems to minimize the required energy and time of the local computing and fog computing modes, where the time assignments and the transmit power were jointly optimized. The closed-form expression of the optimal time assignment for energy harvesting, the closed-form and semi-closed-form expressions of the optimal transmit power and time assignment for offloading were derived by adopting the Lagrangian dual method, KKT conditions and Lambert $\mathcal{W}$ Function. Simulation results showed that, when the sensor has poor computing capacity or when it is far away from the AP, the fog computing should be selected to alleviate the computational burden and save energy for the sensor; otherwise, the local computing mode should be selected to achieve a better performance.

Author Contributions: Y.L. and K.X. equally contributed to this work on system modeling and methodology; K.X. also contributed to project administration; Y.L. also contributed to writing and editing; Y.Z. and L.Z. contributed to the review and editing; and F.L. and T.L. contributed to funding acquisition and simulations.

Funding: This work was supported in part by the National Key R\&D Program of China (No. 2016YFE0200900), in part by the Fundamental Research Funds for the Central Universities (No. 2018YJS040), in part by the Beijing Intelligent Logistics System Collaborative Innovation Center (No. BILSCIC-2018KF-05), in part by the General Program of the National Natural Science Foundation of China (NSFC) (No. 61671051), in part by the major projects of Beijing Municipal Science and Technology Commission (No. Z181100003218010), and also in part by the Young Talents Program of State Grid Energy Research Institute Co., Ltd. (Research on the key technology of fog computing for smart grid, No. XM2018020035180).

Conflicts of Interest: The authors declare no conflict of interest. 


\section{Abbreviations}

The following abbreviations are used in this manuscript:

$\begin{array}{ll}\text { AP } & \text { Access Point } \\ \text { RF } & \text { Radio Frequency } \\ \text { IoT } & \text { Internet of Things } \\ \text { EH } & \text { Energy Harvesting } \\ \text { WPT } & \text { Wireless Power Transfer } \\ \text { WSNs } & \text { Wireless Sensor Networks } \\ \text { WPCNs } & \text { Wireless Powered Communication Networks } \\ \text { SWIPT } & \text { Simultaneous Wireless Information and Power Transfer } \\ \text { PS } & \text { Power Splitting } \\ \text { TS } & \text { Time Switching } \\ \text { ID } & \text { Information Decoding } \\ \text { EE } & \text { Energy Efficient }\end{array}$

\section{References}

1. Hajji, W.; Tso, F. Understanding the Performance of Low Power Raspberry Pi Cloud for Big Data. Electronics 2016, 5, 29. [CrossRef]

2. Bassoli, M.; Bianchi, V.; Munari, I. A Plug and Play IoT Wi-Fi Smart Home System for Human Monitoring. Electronics 2018, 7, 200. [CrossRef]

3. Liu, J.; Zha, L.; Cao, J.; Fei, S. Hybrid-driven-based stabilisation for networked control systems. IET Control Theory Appl. 2016, 10, 2279-2285. [CrossRef]

4. Bangui, H.; Rakrak, S.; Raghay, S.; Buhnova, B. Moving to the Edge-Cloud-of-Things: Recent Advances and Future Research Directions. Electronics 2018, 7, 309. [CrossRef]

5. Cao, J.; Bu, Z.; Gao, G.; Tao, H.; Weighted modularity optimization for crisp and fuzzy community detection in large-scale networks. Physica A 2016, 462, 386-395. [CrossRef]

6. Wu, Z.; Cao, J.; Wang, Y.; Wang, Y.; Zhang, L.; Wu, J. hPSD: A Hybrid PU-Learning-Based Spammer Detection Model for Product Reviews. IEEE Trans. Cybern. 2018. . [CrossRef]

7. Lin, F.; Lu, X.; You, I.; Zhou, X. A Novel Utility Based Resource Management Scheme in Vehicular Social Edge Computing. IEEE Access 2018, 6, 66673-66684. [CrossRef]

8. Lin, F.; Zhou, Y.; Pau, G.; Collotta, M. Optimization-Oriented Resource Allocation Management for Vehicular Fog Computing. IEEE Access 2018, 6, 69294-69303. [CrossRef]

9. Krikidis, I.; Timotheou, S.; Nikolaou, S.; Zheng, G.; Ng, D.; Schober, R. Simultaneous Wireless Information and Power Transfer in Modern Communication Systems. IEEE Commun. Mag. 2014, 52, 104-110. [CrossRef]

10. Ouazir, Y.; Takorabet, N.; Ibtiouen, R.; Mezani, S. Consideration of space harmonics in complex finite element analysis of induction motors with an air-gap interface coupling. IEEE Trans. Magn. 2006, 42, 1279-1282. [CrossRef]

11. Arumugam, D.; Ricketts, D. Passive Magnetoquasistatic Position Measurement Using Coupled Magnetic Resonances. IEEE Antennas Wirel. Propag. Lett. 2013, 12, 539-542. [CrossRef]

12. Lu, Y.; Xiong, K.; Fan, P.; Zhong, Z.; Ben L.K. Coordinated Beamforming with Artificial Noise for Secure SWIPT under Non-Linear EH Model: Centralized and Distributed Designs. IEEE J. Sel. Areas Commun. 2018, 36, 1544-1563. [CrossRef]

13. Lu, Y.; Xiong, K.; Fan, P.; Zhong, Z.; Letaief, K. B. Robust Transmit Beamforming With Artificial Redundant Signals for Secure SWIPT System Under Non-Linear EH Model. IEEE Trans. Wirel. Commun. 2018, 17, 2218-2232. [CrossRef]

14. Liu, X.; Jin, N.; Yang, X.; Hashmi, K.; Ma, D.; Tang, H. A Novel Single-switch Phase Controlled Wireless Power Transfer System. Electronics 2018, 7, 11. [CrossRef]

15. Hu, H.; Xiong, K.; Zhang, Y.; Fan, P.; Liu, T.; Kang, S. Age of Information in Wireless Powered Networks in Low SNR Region for Future 5G. Entropy 2018, 20 , 12. [CrossRef]

16. Xiong, K.; Chen, C.; Qu, G.; Fan, P.; Letaief, K. Group cooperation with optimal resource allocation in wireless powered communication networks. IEEE Trans. Wirel. Commun. 2017, 16, 3840-3853. [CrossRef] 
17. Shi, Q.; Liu, L.; Xu, W.; Zhang, R. Joint transmit beamforming and receive power splitting for MISO SWIPT systems. IEEE Trans. Wirel. Commun. 2014, 13, 3269-3280. [CrossRef]

18. Timotheou, S.; Krikidis, I.; Zheng, G.; Ottersten, B. Beamforming for MISO interference channels with QoS and RF energy transfer. IEEE Trans. Wirel. Commun. 2014, 13, 2646-2658.

19. Shi, Q.; Xu, W.; Chang, T.; Wang, Y.; Song, E. Joint beamforming and power splitting for MISO interference channel with SWIPT: An SOCP relaxation and decentralized algorithm. IEEE Trans. Signal Process. 2014, 62, 6194-6208. [CrossRef]

20. Xiong, K.; Wang, B.; Liu, K. Rate-Energy Region of SWIPT for MIMO Broadcasting under Nonlinear Energy Harvesting Model. IEEE Trans. Wirel. Commun. 2017, 16, 5147-5161. [CrossRef]

21. Lee, S.; Liu, L.; Zhang, R. Collaborative wireless energy and information transfer in interference channel. IEEE Trans. Wirel. Commun. 2015, 14, 545-557. [CrossRef]

22. Zhang, R.; Ho, C.K. MIMO broadcasting for simultaneous wireless information and power transfer. IEEE Trans. Wirel. Commun. 2013, 12, 1989-2001. [CrossRef]

23. Liu, J.; Mao, Y.; Zhang, J.; Letaief, K. Delay-optimal computation task scheduling for mobile-edge computing systems. In Proceedings of the 2016 International Symposium on Information Theory (ISIT), Barcelona, Spain, 10-15 July 2016.

24. Zhang, Y.; Niyato, D.; Wang, P. Offloading in mobile cloudlet systems with intermittent connectivity. IEEE Trans. Mob. Comput. 2015, 14, 2516-2529. [CrossRef]

25. Cao, X.; Wang, F.; Xu, J.; Zhang, R.; Cui, S. Joint Computation and Communication Cooperation for Mobile Edge Computing. In Proceedings of the 2018 16th International Symposium on Modeling and Optimization in Mobile, Ad Hoc, and Wireless Networks, Shanghai, China, 7-11 May 2018.

26. Chang, Z.; Zhou, Z.; Ristaniemi, T.; Niu, Z. Energy Efficient Optimization for Computation Offloading in Fog Computing System. In Proceedings of the 2017 36th Global Communications Conference (GLOBECOM), Singapore, 4-8 December 2017.

27. Wang, F.; Xu, J.; Ding, Z. Optimized Multiuser Computation Offloading with Multi-antenna NOMA. In Proceedings of the 2017 36th Global Communications Conference (GLOBECOM), Singapore, 4-8 December 2017.

28. Ju, H.; Zhang, R. Throughput Maximization in Wireless Powered Communication Networks. IEEE Trans. Wirel. Commun. 2014, 13, 418-428. [CrossRef]

29. Liu, T.; Wang, X.; Zheng, L. A Cooperative SWIPT Scheme for Wirelessly Powered Sensor Networks. IEEE Trans. Wirel. Commun. 2017, 65, 2740-2752. [CrossRef]

30. Wu, D.; Wang, F.; Cao, X.; Xu, J. Wireless Powered User Cooperative Computation in Mobile Edge Computing Systems. airxv 2018, airxv:1809.01430.

31. You, C.; Huang, K.; Chae, H.; Energy Efficient Mobile Cloud Computing Powered by Wireless Energy Transfer. IEEE J. Sel. Areas Commun. 2016, 34, 1757-1771. [CrossRef]

32. Wang, F.; Xu, J.; Wang, X.; Cui, S. Joint Offloading and Computing Optimization in Wireless Powered Mobile-Edge Computing Systems. IEEE Trans. Wirel. Commun. 2017, 17, 1784-1797. [CrossRef]

33. Hu, X.; Wong, K.; Yang, K. Wireless Powered Cooperation-Assisted Mobile Edge Computing. IEEE Trans. Wirel. Commun. 2018, 17, 2375-2388. [CrossRef]

34. Mao, S.; Leng, S.; Yang, K.; Zhao, Q.; Liu, M. Energy Efficiency and Delay Tradeoff in Multi-User Wireless Powered Mobile-Edge Computing Systems. In Proceedings of the 2017 Global Communications Conference (GLOBECOM), Singapore, 4-8 December 2017.

35. Bi, S.; Zhang, Y.; Computation Rate Maximization for Wireless Powered Mobile-Edge Computing With Binary Computation Offloading. IEEE Trans. Wirel. Commun. 2018, 17, 4177-4190. [CrossRef]

36. Zheng, H.; Xiong, K.; Fan, P.; Zhou, L.; Zhong, Z. SWIPT-Aware Fog Information Processing: Local Computing vs. Fog Offloading. Sensors 2018, 18, 3291. [CrossRef]

37. Xiong, K.; Fan, P.; Lu, Y.; Khaled, B. Energy Efficiency with Proportional Rate Fairness in Multi-Relay OFDM Networks. IEEE J. Sel. Areas Commun. 2015, 34, 1431-1447. [CrossRef]

38. Di, X.; Xiong, K.; Fan, P.; Yang, H. Simultaneous Wireless Information and Power Transfer in Cooperative Relay Networks With Rateless Codes. IEEE Trans. Veh. Technol. 2015, 66, 2981-2996. [CrossRef]

39. Meraji, R. Low Power Decoding Circuits for Ultra Portable Devices. Ph.D. Thesis, Lund University, Lund, Sweden, October 2014.

40. Rabaey, J. Low Power Design Essentials; Springer: Berlin, Germany, 2009. 
41. Mammela, A.; Anttonen, A. Why Will Computing Power Need Particular Attention in Future Wireless Devices. IEEE Circuits Syst. Mag. 2017, 17, 12-26. [CrossRef]

42. Boyd, S.P.; Vandenberghe, L. Convex Optimization; Cambridge University Press: Cambridge, UK, 2004.

43. Xiong, K.; Fan, P.; Zhang, Y.; Ben, L. Towards 5G High Mobility: A Fairness-Adjustable Time-Domain Power Allocation Approach. IEEE Access 2017, 5, 11817-11831. [CrossRef]

44. Series, P. Propagation Data and Prediction Methods for the Planning of Indoor Radio Communication Systems and Radio Local Area Networks in the Frequency Range 900 MHz to $100 \mathrm{GHz}$; Recommendation p. 1238-7; International Telecommunication Union: Geneva, Switzerland, 2012.

(C) 2019 by the authors. Licensee MDPI, Basel, Switzerland. This article is an open access article distributed under the terms and conditions of the Creative Commons Attribution (CC BY) license (http:/ / creativecommons.org/licenses/by/4.0/). 